JGG 2022;70:9-16

doi: $10.36150 / 2499-6564-N 261$

\title{
Determinants of physical function in community dwelling old people
}

\author{
Olöf G. Geirsdottir ${ }^{1,2}$, Milan Chang ${ }^{1,3}$, Kristin Briem ${ }^{4}$, Pálmi V. Jonsson ${ }^{1,5}$, \\ Inga Thorsdottir 6 , Alfonso Ramel $l^{1,2}$ \\ ${ }^{1}$ The Icelandic Gerontological Research Center, Reykjavik, Iceland; ${ }^{2}$ Faculty of Food Science \\ and Nutrition, University of Iceland, Reykjavik, Iceland; ${ }^{3}$ Sport Science, School of Science and \\ Engineering, Reykjavik University, Reykjavik, Iceland; ${ }^{4}$ Department of Physiotherapy, University \\ of Iceland, Reykjavik, Iceland; ${ }^{5}$ Department of Geriatrics, National University Hospital of Iceland, \\ Reykjavik, Iceland; ${ }^{6}$ School of Health Sciences, University of Iceland, Reykjavik, Iceland
}

Background. Poor physical function is associated with disability and mortality in old people.

Objectives. The aim was to find determinants of physical function in old people.

Design. Secondary, cross-sectional analysis.

Setting. Community in the Reykjavik, Iceland.

Participants. 236 old people (73.7 \pm 5.7 years, $58.2 \%$ female).

Measurements. Timed-up-and-go (TUG), six-minute-walk-for-distance (6MWD), anthropometrics, quadriceps strength, dietary intake, mini-mental-state-examination (MMSE), leisure-time physical activity (LTPA) and blood variables were assessed. Descriptive, bivariate and multivariate statistical analyses were used.

Results. There were differences between men and women in energy intake, body composition and muscular strength, but physical function did not differ between men and women. In bivariate analysis, most of the assessed variables correlated with 6MWD and TUG. Stepwise linear models showed that age, body composition, strength, medication, LTPA and MMSE were predictors of physical function but not hematological variables. The association between MMSE and function disappeared when corrected for strength/body weight. Results were similar for both 6MWD and TUG and the strongest predictors in the final models were age and quadriceps strength/body weight.

Conclusions. In community dwelling old people, physical function decreases with age. However, it is of clinical relevance that there are modifiable determinants of physical function, in particular strength for a given body weight, LTPA and number of medications, which represent potential targets to maintain physical function in this age group. Our results also indicate that neither cognitive function, nor dietary intake nor blood chemical variables were independently associated with physical function.

Key words: physical function, 6-minute-walk-for-distance, timed-up-

and-go, community dwelling old people

\section{INTRODUCTION}

There is convincing evidence that poor physical function is associated with adverse health outcomes in old people ${ }^{1-4}$, e.g., it has been reported that \footnotetext{
dance with the CC-BY-NC-ND (Creative Commons Attribution-NonCommercial-NoDerivatives 4.0 International) license. The article can be used by giving appropriate credit and mentioning the license, but original version. For further information: https://creativecommons.org/licenses/by-nc-nd/4.0/deed.en
} \\ This is an open access article distributed in accor- \\ only for non-commercial purposes and only in the \\ (C) Copyright by Società Italiana
di Gerontologia e Geriatria (SIGG) \\ OPEN ACCESS \\ How to cite this article: Geirsdottir OG, \\ physical function in community dwelling \\ old people. Journal of Gerontology and \\ Geriatrics 2022;70:9-16. https://doi.
org/10.36150/2499-6564-N261 \\ Received: September 3, 2020 \\ Alfons Ramel \\ Faculty of Food Science and Nutrition, University \\ Tel. +354 543 9875. Fax +354 5434824 \\ E-mail: alfonsra@hi.is
}


physical function is associated with disability in both cross-sectional and longitudinal studies ${ }^{1,2}$. Further, poor function is associated with greater hazards of injurious falls ${ }^{3}$ and according to a longitudinal study, physical function predicts mortality in community dwelling old people even after correction for various confounders ${ }^{4}$. Several studies are available that have characterized old people with poor physical function ${ }^{5-18}$. Nutrition status, physical activity and body composition have all been associated with physical function ${ }^{5,6}$. One study reported that calf circumference was positively related to higher functional performance in old people ${ }^{7}$. Muscle quality and relative adiposity were strong and independent predictors of physical function in older women ${ }^{8}$, although findings from other studies have been inconsistent ${ }^{9,10}$.

Blood chemical variables have also been associated with physical function in old people, e.g., hyperuricemia was associated with poor physical performance in older people, over a follow-up of 4.4 years ${ }^{11}$. Further, chronic inflammation has been proposed as a biological mechanism underlying the decline in physical function that occurs with aging ${ }^{12}$. In a recently published longitudinal study, both IL-6 and CRP had some associations with physical performance at baseline, although they did not predict changes in performance seven years later in older adults ${ }^{12}$.

In recent years, numerous studies have investigated physical fitness and cognitive impairment in older people in relation to ageing ${ }^{13,14}$, but only a limited number of studies have focused on the association between physical and cognitive function ${ }^{15}$. As such, a recently published study found associations between physical function and cognitive impairment in people over 65 years old. Specifically, results indicated that gait speed is the variable that best represents both the cognitive and physical function in people over 65 years of age ${ }^{15}$. Medications have also been associated with poor physical function and old people with poor physical function tend to use a higher number of medications ${ }^{16}$ and pharmacologic burden is significantly and independently associated with falls in that group ${ }^{17}$.

Obviously, a large number of variables can be associated with physical function. As many of the above mentioned studies focus mainly on one or few of these factors, and as there is plenty of potential interaction between them, it is not known which variables are independently associated with physical function. Thus, the of the aim of the present study was to find determinants of physical function in old people and to investigate whether body composition and muscular strength can explain observed associations of physical function with cognitive function, blood chemical variables, dietary intake and medication.
This was a secondary data analysis from baseline data of a previously published randomized, controlled trial, designed to examine the effect of post-exercise protein ingestion on the efficacy of strength training in old people ${ }^{18}$.

\section{METHODS}

\section{SUBJECTS}

Participants ( $N=236$ ) were 65 years and older (range 65-92 years old) and were recruited by advertisements posted in the Reykjavik area. Exclusion criteria were low cognitive function (Mini-Mental State Examination (MMSE) $\leq 19$ points) ${ }^{19}$, major orthopedic disease and pharmacological interventions with exogenous testosterone or other drugs known to influence muscle mass. Furthermore, participants had to be free of any musculoskeletal disorders or other disorders that could affect their muscle mass. Enrolled subjects were apparently healthy, although some had hypertension, hyperlipidemia or type 2 diabetes ${ }^{19,20}$. The study was approved by the Icelandic National Bioethics Committee (15-139-S1) and has therefore been performed in accordance with the ethical standards laid down in the 1964 Declaration of Helsinki and its later amendments. All persons gave their informed consent prior to their inclusion in the study.

\section{BODY COMPOSITION}

Body composition (lean body mass = LBM, fat mass $=\mathrm{FM}$, appendicular skeletal muscle $=$ ASM) was assessed by dual energy $\mathrm{x}$-ray absorptiometer (DXA) with Hologic QDR-2000 plus ${ }^{\circledR}$, Hologic Inc., Waltham, MA, USA. The DXA measurements were conducted at the Icelandic Heart Association, Kopavogur, Iceland. Body weight (BW) was measured in light underwear on a calibrated scale (model no. 708, Seca, Hamburg, Germany) and height was measured with a calibrated stadiometer (model no. 206; Seca, Hamburg, Germany). Body mass index (BMI) was calculated from the recorded height and weight $\left(\mathrm{kg} / \mathrm{m}^{2}\right)$. Waist circumference was measured halfway between the top of the lateral iliac crest and the lowest rib. All measures were performed twice using a tape measure and recorded to the nearest centimeter.

\section{MusculAR STRENGTH}

\section{Quadriceps strength}

Quadriceps strength (maximum voluntary isometric contraction (MVIC)) was tested with an isokinetic dynamometer (Kin-Com ${ }^{\circledR} 500 \mathrm{H}$ Chattanooga). The participants performed three submaximal trials and then 
four MVIC tests for five seconds each, with a 50 second rest between tests. The greatest output was recorded as the peak force expressed in Newton (N).

\section{Physical function}

\section{Six Minute Walk for Distance (6MWD)}

Gait speed was assessed with 6MWD. The 6MWD was performed indoors in a spacious gym hall and conducted according to the guidelines from the American Thoracic Society ${ }^{21}$.

\section{Timed Up and Go test (TUG)}

During the TUG test the subject was instructed to rise from a chair with a seat height of $43 \mathrm{~cm}$, walk $3 \mathrm{~m}$, turn around, return and sit down again, wearing ordinary footwear and using customary walking aids if necessary ${ }^{22}$.

\section{QuestionnaIRES}

Demographic characteristics and medication count were collected using questionnaires.

\section{DieTARY ASSESSMENT}

Diet was assessed using a 3-day weighed food record at the start of the study. Participants weighed and recorded their food intake for three consecutive days, two week days and one weekend day. Instructions on how to record the diet were given orally and in writing. The participants were provided with electronic scales (PHILIPS HR 2393) and were asked to record all food items and drinks. The results of the food records were typed into an online food calculation program based on the ISGEM databank, which contains data on the composition of foods on the Icelandic market.

\section{LEISURE-TIME PHYSICAL ACTIVITY (LTPA)}

Information on LTPA during the last year was collected using a questionnaire ${ }^{23}$ based on the Compendium of Physical Activities ${ }^{24}$ and the Paffenberger's questionnaire ${ }^{25}$. The LTPA for each subject was evaluated by asking them to report their participation in sports, exercises or other physical activities as time per week. In statistical analysis it is shown as hours/week.

\section{BIOCHEMICAL MEASUREMENTS}

Participants were instructed to avoid strenuous exercise and alcohol consumption the day before the drawing of fasting blood samples at baseline and endpoint. The blood samples were centrifuged and the serum was stored at $-80^{\circ} \mathrm{C}$ for subsequent analysis at the University Hospital in Reykjavik, Iceland. Glucose was analyzed using an enzymatic colorimetric assay and an automated analyzer. HbA1c was measured using a chromatographic-spectrophotometric assay. C-reactive protein was measured with ELISA.

Table I. Characteristics of male and female participants.

\begin{tabular}{|c|c|c|c|c|c|c|c|c|c|c|}
\hline \multirow[b]{2}{*}{ Age (years) } & \multicolumn{3}{|c|}{$\begin{array}{c}\text { All } \\
(\mathrm{n}=236) \\
\text { Mean } \pm \text { SD }\end{array}$} & \multicolumn{3}{|c|}{$\begin{array}{c}\text { Male } \\
(n=98) \\
\text { Mean } \pm \text { SD }\end{array}$} & \multicolumn{3}{|c|}{$\begin{array}{c}\text { Female } \\
(n=138) \\
\text { Mean } \pm \text { SD }\end{array}$} & \multirow{2}{*}{\begin{tabular}{|c|} 
P-value \\
0.018 \\
\end{tabular}} \\
\hline & 73.6 & \pm & 5.7 & 74.6 & \pm & 5.9 & 72.8 & \pm & 5.5 & \\
\hline Height (cm) & 169 & \pm & 10 & 178 & \pm & 8 & 163 & \pm & 6 & $<0.001$ \\
\hline Body weight $(\mathrm{kg})$ & 82.6 & \pm & 17.5 & 93.9 & \pm & 16.7 & 74.6 & \pm & 13.1 & $<0.001$ \\
\hline Waist circumference (cm) & 99.8 & \pm & 14.4 & 108.5 & \pm & 12.1 & 93.6 & \pm & 12.6 & $<0.001$ \\
\hline $\mathrm{BMI}\left(\mathrm{kg} / \mathrm{m}^{2}\right)$ & 28.8 & \pm & 4.8 & 29.7 & \pm & 4.6 & 28.1 & \pm & 4.9 & 0.012 \\
\hline Fat mass $(\mathrm{kg})$ & 38.2 & \pm & 7.3 & 32.3 & \pm & 10.4 & 31.4 & \pm & 9.5 & 0.515 \\
\hline Appendicular skeletal muscle (kg) & 24.4 & \pm & 5.4 & 29.6 & \pm & 3.9 & 20.9 & \pm & 2.6 & $<0.001$ \\
\hline Quadriceps strength (N) & 464 & \pm & 124 & 538 & \pm & 124 & 409 & \pm & 90 & $<0.001$ \\
\hline 6MWD (m) & 453 & \pm & 80 & 456 & \pm & 86 & 452 & \pm & 76 & 0.708 \\
\hline TUG (sec) & 7.9 & \pm & 2.2 & 8.0 & \pm & 2.1 & 7.9 & \pm & 2.3 & 0.595 \\
\hline $\mathrm{CRP}(\mathrm{mg} / \mathrm{L})$ & 7.1 & \pm & 4.6 & 7.4 & \pm & 5.4 & 6.9 & \pm & 4.0 & 0.487 \\
\hline Glucose (mmol/L) & 5.0 & \pm & 1.0 & 5.2 & \pm & 1.1 & 4.8 & \pm & 0.9 & 0.020 \\
\hline $\mathrm{HbA1c}(\%)$ & 5.7 & \pm & 0.6 & 5.8 & \pm & 0.7 & 5.6 & \pm & 0.5 & 0.014 \\
\hline MMSE (score) & 27.5 & \pm & 2.1 & 27.1 & \pm & 2.1 & 27.8 & \pm & 2.0 & 0.021 \\
\hline Number of drugs & 2.1 & \pm & 1.5 & 2.3 & \pm & 1.4 & 1.9 & \pm & 1.6 & 0.067 \\
\hline LTPA** $^{* *}$ /week) & 342 & \pm & 342 & 323 & \pm & 341 & 356 & \pm & 343 & 0.462 \\
\hline Energy intake (kcal/d) & 1679 & \pm & 481 & 1886 & \pm & 528 & 1531 & \pm & 382 & $<0.001$ \\
\hline Protein intake (g/kg BW/d) & 0.95 & \pm & 0.27 & 0.96 & \pm & 0.26 & 0.94 & \pm & 0.28 & 0.587 \\
\hline
\end{tabular}

*Differences between genders according to an independent samples t test (normally distributed variables) and Mann Whitney U test (not normally distributed variables); ${ }^{\star \star}$ Leisure time physical activity 
Table II. Correlation table*.

\begin{tabular}{|c|c|c|c|}
\hline \multicolumn{2}{|c|}{ Variables } & Pearson r & P-value \\
\hline \multirow[t]{2}{*}{ Age (years) } & 6MWD & -0.502 & $<0.001$ \\
\hline & TUG (sec) & 0.508 & $<0.001$ \\
\hline \multirow[t]{2}{*}{ BMI $\left(\mathrm{kg} / \mathrm{m}^{2}\right)$} & 6MWD & -0.245 & $<0.001$ \\
\hline & TUG (sec) & 0.151 & 0.023 \\
\hline \multirow[t]{2}{*}{ Fat mass $(\mathrm{kg})$} & 6MWD & -0.238 & $<0.001$ \\
\hline & TUG (sec) & 0.139 & 0.040 \\
\hline \multirow[t]{2}{*}{ Lean body mass $(\mathrm{kg})$} & 6MWD & 0.037 & 0.581 \\
\hline & TUG (sec) & 0.002 & 0.979 \\
\hline \multirow[t]{2}{*}{ ASM (kg) } & $6 \mathrm{MWD}$ & 0.077 & 0.245 \\
\hline & TUG (sec) & -0.039 & 0.562 \\
\hline \multirow[t]{2}{*}{ ASM (\%) } & 6MWD & 0.328 & $<0.001$ \\
\hline & TUG (sec) & -0.219 & 0.001 \\
\hline \multirow[t]{2}{*}{ Quadriceps strength (N) } & 6MWD & 0.416 & $<0.001$ \\
\hline & TUG (sec) & -0.365 & $<0.001$ \\
\hline \multirow[t]{2}{*}{ Quadriceps strength/body weight (N/kg) } & 6MWD & 0.537 & $<0.001$ \\
\hline & TUG (sec) & -0.480 & $<0.001$ \\
\hline \multirow[t]{2}{*}{ HbA1c (\%) } & $6 \mathrm{MWD}$ & -0.246 & $<0.001$ \\
\hline & TUG (sec) & 0.224 & 0.001 \\
\hline \multirow[t]{2}{*}{$\mathrm{CRP}(\mathrm{mg} / \mathrm{L})$} & 6MWD & -0.155 & 0.018 \\
\hline & TUG (sec) & 0.050 & 0.454 \\
\hline \multirow[t]{2}{*}{ MMSE (score) } & 6MWD & 0.400 & $<0.001$ \\
\hline & TUG (sec) & -0.431 & $<0.001$ \\
\hline \multirow[t]{2}{*}{ Number of medications } & 6MWD & -0.303 & $<0.001$ \\
\hline & TUG (sec) & 0.250 & $<0.001$ \\
\hline \multirow[t]{2}{*}{ Physical activity (h/week) } & 6MWD & 0.364 & $<0.001$ \\
\hline & TUG (sec) & -0.296 & $<0.001$ \\
\hline \multirow{2}{*}{ Energy intake (kcal/day) } & 6MWD & 0.039 & 0.585 \\
\hline & TUG (sec) & -0.007 & 0.919 \\
\hline \multirow[t]{2}{*}{ Protein intake (g/d/body weight) } & 6MWD & 0.086 & 0.223 \\
\hline & TUG (sec) & -0.127 & 0.072 \\
\hline
\end{tabular}

*Spearman 's correlation coefficient rho.

\section{Statistical ANALYSIS}

Statistical analysis was conducted using SPSS for Windows version 22.0 (SPSS, Chicago, IL, USA) and the level of significance was set at $P<0.05$. Data were checked for normality using the Kolmogorov-Smirnov test and are shown as mean \pm standard deviation (SD). Comparisons between groups, e.g., men and women, were done using independent samples' t-test (normally distributed variables) or Mann-Whitney- $U$ test (not normally distributed variables). Correlations between variables were calculated using Spearman 's correlation coefficient rho. Linear models with various degrees of statistical correction were used to find variables associated with 6MWD and TUG. Model 1 included sex, age and factors related to chronic conditions; model 2 additionally included lifestyle factors; model 3 additionally body composition; model 4 additionally included muscular strength.

\section{RESULTS}

Baseline data and differences between genders can be seen in Table I. Not unexpected, there were differences between men and women in energy intake, body composition and muscular strength. However, physical function (6MWD, TUG) did not differ between men and women.

Table II shows correlations of physical function with body composition, strength, LTPA, medication, hematological variables, MMSE, energy and protein intake. With the exception of CRP, variables that were significantly associated with 6MWD and TUG were identical. The multivariate linear models in Table III and IV show that age, number of medications and habitual physical activity are consistently associated with physical function rather independently from other covariates. Hematological variables were not associated with physical function in the models and the association between gender and MMSE 
Table III. Determinants* of 6MWD (m).

\begin{tabular}{|c|c|c|c|c|c|c|c|c|c|c|c|c|c|c|c|c|}
\hline \multirow[b]{3}{*}{ Intercept } & \multicolumn{4}{|c|}{$\begin{array}{c}\text { Model } 1 \\
\text { Age, gender + chronic } \\
\text { conditions }\end{array}$} & \multicolumn{4}{|c|}{$\begin{array}{c}\text { Model } 2 \\
\text { Additional: lifestyle }\end{array}$} & \multicolumn{4}{|c|}{$\begin{array}{c}\text { Model } 3 \\
\text { Additional: body } \\
\text { composition }\end{array}$} & \multicolumn{4}{|c|}{$\begin{array}{c}\text { Model } \mathbf{4} \\
\text { Additional: strength }\end{array}$} \\
\hline & \multirow{2}{*}{\begin{tabular}{|l|} 
B \\
674
\end{tabular}} & \multicolumn{2}{|c|}{$95 \% \mathrm{CI}$} & \multirow{2}{*}{\begin{tabular}{|l|} 
P-value \\
$<0.001$
\end{tabular}} & \multirow{2}{*}{$\begin{array}{l}\text { B } \\
666\end{array}$} & \multicolumn{2}{|c|}{$95 \% \mathrm{CI}$} & \multirow{2}{*}{\begin{tabular}{|l|} 
P-value \\
$<0.001$ \\
\end{tabular}} & \multirow{2}{*}{$\begin{array}{l}\text { B } \\
623\end{array}$} & \multicolumn{2}{|c|}{$95 \% \mathrm{Cl}$} & \multirow{2}{*}{$\begin{array}{l}\text { P-value } \\
<0.001\end{array}$} & \multirow{2}{*}{$\begin{array}{l}\text { B } \\
629\end{array}$} & \multicolumn{2}{|c|}{$95 \% \mathrm{CI}$} & \multirow{2}{*}{$\begin{array}{l}\text { P-value } \\
<0.001\end{array}$} \\
\hline & & 466 & 882 & & & 462 & 869 & & & 376 & 869 & & & 382 & 876 & \\
\hline $\begin{array}{c}\text { Age } \\
\text { (years) }\end{array}$ & -5.3 & -6.9 & \begin{tabular}{|l|}
-3.7 \\
\end{tabular} & $<0.001$ & -5.0 & -6.5 & -3.4 & $<0.001$ & -5.7 & -7.2 & -4.1 & $<0.001$ & -5.1 & -6.6 & -3.6 & $<0.001$ \\
\hline Male** & 23.7 & 6.7 & 40.8 & 0.007 & 22.5 & 5.9 & 39.2 & 0.008 & 7.7 & -16.8 & 32.2 & 0.535 & 9.5 & -13.7 & 32.7 & 0.421 \\
\hline HbA1c (\%) & -12.7 & -26.8 & 1.3 & 0.075 & -11.0 & -24.8 & 2.7 & 0.115 & -5.2 & -18.5 & 8.2 & 0.446 & -1.2 & -13.9 & 11.5 & 0.851 \\
\hline $\begin{array}{c}\mathrm{CRP} \\
(\mathrm{mg} / \mathrm{L})\end{array}$ & -0.8 & -2.6 & 1.0 & 0.394 & -0.5 & -2.3 & 1.3 & 0.571 & -0.4 & -2.1 & 1.4 & 0.689 & -1.1 & -2.8 & 0.6 & 0.198 \\
\hline $\begin{array}{l}\text { MMSE } \\
\text { (score) }\end{array}$ & 9.3 & 5.0 & 13.6 & $<0.001$ & 7.4 & 3.0 & 11.9 & 0.001 & 5.8 & 1.4 & 10.3 & 0.010 & 1.8 & -2.5 & 6.2 & 0.414 \\
\hline $\begin{array}{c}\text { Number of } \\
\text { drugs }\end{array}$ & -8.3 & -14.1 & -2.5 & 0.005 & -7.3 & -12.9 & -1.6 & 0.012 & -7.1 & -12.6 & -1.6 & 0.012 & -8.1 & -13.3 & -2.9 & 0.003 \\
\hline $\begin{array}{l}\text { Smoking } \\
\text { (yes) }\end{array}$ & & & & & -17.3 & -52.2 & 17.6 & 0.330 & -14.6 & -47.7 & 18.4 & 0.383 & 3.0 & -30.0 & 35.9 & 0.859 \\
\hline $\begin{array}{c}\text { Alcohol } \\
\text { (yes) }\end{array}$ & & & & & 7.1 & \begin{tabular}{|l|}
-14.9 \\
\end{tabular} & 29.0 & 0.527 & 5.4 & -15.6 & 26.3 & 0.615 & 0.0 & -20.5 & 20.4 & 0.997 \\
\hline $\begin{array}{c}\text { Physical } \\
\text { activity (h/ } \\
\text { week) }\end{array}$ & & & & & 3.1 & 1.7 & 4.6 & $<0.001$ & 2.2 & 0.8 & 3.6 & 0.002 & 2.2 & 0.8 & 3.5 & 0.001 \\
\hline & & & & & & & & & & & & & & & & \\
\hline $\begin{array}{c}\text { Fat mass } \\
(\mathrm{kg})\end{array}$ & & & & & & & & & -0.9 & -2.2 & 0.4 & 0.162 & -0.2 & -1.4 & 1.1 & 0.789 \\
\hline ASM (\%) & & & & & & & & & 4.88 & 0.44 & 9.32 & 0.031 & 2.68 & -1.58 & 6.95 & 0.216 \\
\hline $\begin{array}{c}\text { Quadr. } \\
\text { strength/ } \\
\text { BW }(\mathrm{N} / \mathrm{kg})\end{array}$ & & & & & & & & & & & & & 16.2 & 9.0 & 23.5 & $<0.001$ \\
\hline
\end{tabular}

*using linear models; **as opposed to female

with physical function disappear after correction for body composition and strength. The results also indicate that fat mass per se is not associated with function, but ASM is. Results were similar for both 6MWD and TUG and the strongest variables in the final models were age (eta squared $=18$ and $21 \%$, respectively) and strength/body weight (eta squared $=10$ and $8 \%$, respectively).

\section{DISCUSSION}

The present study aimed to find determinants of physical function in community dwelling old people. Not unexpected, age was the variable strongest related to physical function, however, we found a number of other associated variables that were modifiable. Given the importance of physical function in old people ${ }^{1-4}$, these variables represent potential targets for future interventions.

In our study LTPA, body composition and muscular strength were related to physical function. According to the multivariate models, a strong association between lower extremity strength per kg body weight and lower extremity function was observed. In order to investigate causality or the direction of this relation, increasing strength and/or reducing body weight could represent the target for future longitudinal, interventional studies. Others have reported similar results, i.e., that muscle quality (strength for a given amount of lean mass) rather than absolute lean mass is the most important predictor of function ${ }^{8}$. Interestingly, in the statistical analysis LTPA remained significantly associated with physical function although we corrected for body composition and strength. This might potentially be related to better 
Table IV. Determinants* of TUG (sec).

\begin{tabular}{|c|c|c|c|c|c|c|c|c|c|c|c|c|c|c|c|c|}
\hline \multirow[b]{3}{*}{ Intercept } & \multicolumn{4}{|c|}{$\begin{array}{c}\text { Model 1 } \\
\text { Age, gender + chronic } \\
\text { conditions }\end{array}$} & \multicolumn{4}{|c|}{$\begin{array}{c}\text { Model } 2 \\
\text { Additional: lifestyle }\end{array}$} & \multicolumn{4}{|c|}{$\begin{array}{c}\text { Model } 3 \\
\text { Additional: body } \\
\text { composition }\end{array}$} & \multicolumn{4}{|c|}{$\begin{array}{c}\text { Model } 4 \\
\text { Additional: strength }\end{array}$} \\
\hline & \multirow{2}{*}{\begin{tabular}{|l|} 
B \\
3.08 \\
\end{tabular}} & \multicolumn{2}{|c|}{$95 \%$ Cl } & \multirow{2}{*}{\begin{tabular}{|l|} 
P-value \\
0.310 \\
\end{tabular}} & \multirow{2}{*}{$\begin{array}{l}\text { B } \\
2.72\end{array}$} & \multicolumn{2}{|c|}{ 95\% CI } & \multirow{2}{*}{$\begin{array}{c}\text { P-value } \\
0.365 \\
\end{array}$} & \multirow{2}{*}{\begin{tabular}{|l|} 
B \\
4.66 \\
\end{tabular}} & \multicolumn{2}{|c|}{$95 \%$ Cl } & \multirow{2}{*}{$\begin{array}{c}\text { P-value } \\
0.221\end{array}$} & \multirow{2}{*}{$\begin{array}{l}\text { B } \\
1.44 \\
\end{array}$} & \multicolumn{2}{|c|}{$95 \%$ CI } & \multirow{2}{*}{$\begin{array}{r}\text { P-value } \\
0.683\end{array}$} \\
\hline & & -2.88 & 9.05 & & & $\mid-3.19$ & 8.63 & & & -2.83 & 12.15 & & & $\mid-5.51$ & 8.38 & \\
\hline $\begin{array}{c}\text { Age } \\
\text { (years) }\end{array}$ & 0.15 & 0.10 & 0.20 & $<0.001$ & 0.15 & \begin{tabular}{|l|}
0.10 \\
\end{tabular} & 0.19 & $<0.001$ & 0.16 & 0.11 & 0.21 & $<0.001$ & \begin{tabular}{|l|}
0.16 \\
\end{tabular} & \begin{tabular}{|l|}
0.11 \\
\end{tabular} & 0.20 & $<0.001$ \\
\hline $\mathrm{male}^{\star \star}$ & -0.41 & -0.90 & 0.08 & 0.099 & -0.36 & \begin{tabular}{|l|}
-0.84 \\
\end{tabular} & 0.13 & 0.146 & 0.10 & -0.64 & 0.83 & 0.796 & \begin{tabular}{|l|}
0.07 \\
\end{tabular} & $\mid-0.57$ & 0.72 & 0.823 \\
\hline $\begin{array}{c}\mathrm{HbA1c} \\
(\%)\end{array}$ & 0.29 & -0.11 & 0.68 & 0.159 & 0.23 & \begin{tabular}{|l|}
-0.17 \\
\end{tabular} & \begin{tabular}{|l|}
0.62 \\
\end{tabular} & 0.263 & 0.10 & -0.30 & 0.50 & 0.630 & -0.02 & -0.38 & 0.33 & 0.897 \\
\hline $\begin{array}{c}\mathrm{CRP} \\
(\mathrm{mg} / \mathrm{L})\end{array}$ & -0.02 & \begin{tabular}{|l|} 
\\
\end{tabular} & 0.03 & 0.411 & \begin{tabular}{|l|} 
\\
\end{tabular} & \begin{tabular}{|l|}
-0.08 \\
\end{tabular} & \begin{tabular}{|l|}
0.02 \\
\end{tabular} & 0.271 & \begin{tabular}{|l|} 
\\
\end{tabular} & -0.09 & \begin{tabular}{|l|}
0.02 \\
\end{tabular} & 0.167 & \begin{tabular}{|l|} 
\\
\end{tabular} & \begin{tabular}{|l|}
-0.06 \\
\end{tabular} & 0.03 & 0.446 \\
\hline $\begin{array}{l}\text { MMSE } \\
\text { (score) }\end{array}$ & -0.28 & -0.41 & -0.16 & $<0.001$ & -0.23 & \begin{tabular}{|l|}
-0.36 \\
\end{tabular} & -0.10 & 0.001 & -0.20 & $\mid-0.33$ & \begin{tabular}{|l|}
-0.06 \\
\end{tabular} & 0.004 & -0.05 & \begin{tabular}{|l|}
-0.17 \\
\end{tabular} & 0.07 & 0.417 \\
\hline \begin{tabular}{|l|} 
Number \\
of drugs \\
\end{tabular} & 0.16 & -0.01 & 0.32 & 0.063 & 0.14 & \begin{tabular}{|l|}
-0.03 \\
\end{tabular} & \begin{tabular}{|l|}
0.30 \\
\end{tabular} & 0.102 & 0.14 & \begin{tabular}{|l|}
-0.02 \\
\end{tabular} & 0.30 & 0.095 & 0.17 & \begin{tabular}{|l|}
0.03 \\
\end{tabular} & 0.32 & 0.021 \\
\hline $\begin{array}{c}\text { Smoking } \\
\text { (yes) }\end{array}$ & & & & & 1.11 & 0.11 & 2.11 & 0.030 & 1.06 & 0.07 & 2.05 & 0.036 & -0.02 & \begin{tabular}{|l|}
-0.94 \\
\end{tabular} & 0.89 & 0.964 \\
\hline \begin{tabular}{|c|} 
Alcohol \\
(yes)
\end{tabular} & & & & & -0.24 & \begin{tabular}{|l|}
-0.88 \\
\end{tabular} & \begin{tabular}{|l|}
0.40 \\
\end{tabular} & 0.467 & -0.15 & -0.79 & 0.49 & 0.647 & \begin{tabular}{|l|}
0.06 \\
\end{tabular} & -0.51 & 0.64 & 0.828 \\
\hline \begin{tabular}{|c|} 
Physical \\
activity \\
(h/week) \\
\end{tabular} & & & & & -0.06 & \begin{tabular}{|l|}
-0.10 \\
\end{tabular} & -0.02 & 0.005 & -0.04 & \begin{tabular}{|l|}
-0.09 \\
\end{tabular} & 0.00 & 0.042 & -0.04 & \begin{tabular}{|l|}
-0.08 \\
\end{tabular} & -0.01 & 0.024 \\
\hline $\begin{array}{c}\text { Fat mass } \\
(\mathrm{kg})\end{array}$ & & & & & & & & & 0.01 & \begin{tabular}{|l|}
-0.03 \\
\end{tabular} & 0.05 & 0.611 & 0.00 & $\mid-0.04$ & 0.04 & 0.981 \\
\hline ASM (\%) & & & & & & & & & -0.12 & -0.26 & \begin{tabular}{|l|}
1.09 \\
\end{tabular} & 0.071 & -0.41 & \begin{tabular}{|l|}
-0.16 \\
\end{tabular} & 0.08 & 0.495 \\
\hline \begin{tabular}{|c|} 
Quadr. \\
strength/ \\
BW (N/ \\
kg)
\end{tabular} & & & & & & & & & & & & & -0.41 & $\mid-0.61$ & -0.20 & $<0.001$ \\
\hline
\end{tabular}

*using linear models; **as opposed to female

balance of physically active people, another important components of physical function ${ }^{26}$.

We found a significant negative association between the number of medications and physical function, although median medication count was low or around 2 in our participants. With each additional drug 6MWD decreased by around $7 \mathrm{~m}$ and TUG increased by around $0.15 \mathrm{sec}$ according to the statistical model. Similar associations have been reported previously in humans ${ }^{16}$. These associations were independent from body composition or muscular strength, as effect size B did hardly change after correction for fat mass, ASM or quadriceps strengths. However, our study cannot answer whether the drugs were directly associated with lower physical health or if the number of drugs taken by a participant were a proxy of his/her overall health, because the number of chronic diseases were not recorded in the present study.

In a recently published study cognitive function has been reported to be associated with physical function ${ }^{15}$. In our study we assessed cognitive function using the simple screening tool MMSE. We found that MMSE score was related to both TUG and 6MWD, although the distribution of MMSE score was limited due to inclusion criteria in our study. In the multivariate analysis, it turned out that the relationship between MMSE and function was largely explained by muscular strength. In a separate analysis (data not shown) we found that the lowest MMSE quartile had significantly lower quadriceps strength corrected for body weight than the highest quartile. A similar difference between MMSE quartiles in ASM was not seen. Accordingly, results from a recent review on this topic suggest an influence of cognitive function on the muscular strength of old people, which can, according to the authors, affect aspects of their functional capacity and therefore agree with our results ${ }^{27}$.

Poorer physical function has been reported in women compared with men ${ }^{28}$, which was consequently explained by their higher fat mass, but also by other body composition differences. Although we also found sex specific 
differences in body composition and in lower extremity muscle strength, these differences did not translate into poorer lower extremity function in women according to descriptive statistics. Taking into consideration quadriceps strength for each $\mathrm{kg}$ of body weight, the numbers were similar for men $(5.7 \mathrm{~N} / \mathrm{kg})$ and women $(5.5 \mathrm{~N} / \mathrm{kg})$, potentially explaining why we observed similar function. However, as men were significant older than women in our study, this age difference could affect the comparison between genders. In a simple age corrected comparison (data not shown), there was a tendency for better 6MVD in men compared to women $(P=0.08)$, however there were no differences observed for TUG. This can be explained by greater height (leg length) in men, which matters less in the $3 \mathrm{~m}$ TUG distance, but each step (stride length) starts to matter in a 6MWD in which a tall person will walk further than a short person given the same cadence.

Energy intake, protein intake and blood chemical variables were not associated with physical function in study participants. Although energy and protein intake play undoubtly an important role in the maintenance of lean body mass ${ }^{29}$, their associations with physical function are difficult to confirm. Assessment of dietary intake is difficult, especially in old people and the quality of food records depends on the motivation and cognitive function of the old participant ${ }^{30}$. Further, inclusion of lean body mass in statistical analysis will most likely make any potential association between dietary intake and function disappear. Blood chemical variables were associated with function in bivariate analysis, but these associations disappeared in multivariate analysis. Several studies have proposed how deteriorated blood chemical variables can explain poor function ${ }^{11,12}$, but our results indicate that they are not independently associated with physical function.

\section{STRENGTH AND LIMITATIONS}

It is a strength of this study, that information on many potential variables related to physical function was available, which exceeds previously published studies on this topic. This made it possible to gain a wider understanding on which variables were independently associated with physical function. However, this was a cross-sectional study and it lies in the nature of such study design that it can not differentiate between cause and consequence of an observed association. Longitudinal and intervention studies have to confirm present findings.

\section{CONCLUSIONS}

In community dwelling old people, physical function decreases with age. However, there are modifiable determinants of physical function, in particular strength for a given body weight, LTPA and number of medications, which might give the possibility to maintain or improve physical function in this age group. Our results also indicate that neither cognitive function, nor dietary intake nor blood chemical variables were independently associated with physical function.

\section{Ethical consideration}

This manuscript is based on a study which was classified as a low risk investigation because the intervention was supervised physical activity with minimal invasive blood sampling. These ethical considerations are based on the decission from the Icelandic National Bioethics Committee (15-139-S1).

\section{Acknowledgement}

The trial is registered at the US National Library of Medicine (No. NCT01074879).

\section{Funding}

This study was part of the IceProQualita project, which was funded by the Icelandic Technology Development Fund (No 071323008), Research Fund of the University of Iceland, a grant from Landspitali University Hospital Research Fund and the Helga Jonsdottir and Sigurlidi Kristjansson Geriatric Research Fund.

\section{Conflict of interest}

The Authors declare no conflict of interest.

\section{References}

1 Winchester F, Ellis R, Kosma M, et al. Predictors of ADL disability in culturally diverse older adults. Int J Exerc Sci 2009;2:202-214.

2 Artaud F, Singh-Manoux A, Dugravot A, et al. Decline in fast gait speed as a predictor of disability in older adults. J Am Geriatr Soc 2015;63:1129-1136. https://doi.org/10.1111/ jgs.13442

3 Ward RE, Leveille SG, Beauchamp MK, et al. Functional performance as a predictor of injurious falls in older adults. J Am Geriatr Soc 2015;63:315-320. https://doi. org/10.1111/jgs.13203

4 Cesari M, Onder G, Zamboni V, et al. Physical function and self-rated health status as predictors of mortality: results from longitudinal analysis in the iISIRENTE study. BMC Geriatr 2008;8:34. https://doi.org/10.1186/1471-2318-8-34

5 Brady AO, Straight CR, Evans EM. Body composition, muscle capacity, and physical function in older adults: an integrated conceptual model. J Aging Phys Act 2014;22:441 452. https://doi.org/10.1123/japa.2013-0009 
6 Moreira NC, Krausch-Hofmann S, Matthys C, et al. Risk factors for malnutrition in older adults: a systematic review of the literature based on longitudinal data. Adv Nutr 2016;7:507-522. https://doi.org/10.3945/an.115.011254

7 Landi F, Onder G, Russo A, et al. Calf circumference, frailty and physical performance among older adults living in the community. Clin Nutr 2014;33:539-544. https://doi. org/10.1016/j.clnu.2013.07.013

8 Straight CR, Brady AO, Evans EM. Muscle quality and relative adiposity are the strongest predictors of lower-extremity physical function in older women. Maturitas 2015;80:9599. https://doi.org/10.1016/j.maturitas.2014.10.006

9 Fantin F, Di Francesco V, Fontana G, et al. Longitudinal body composition changes in oldmen and women: interrelationships with worsening disability. J Gerontol A: Biol Sci Med Sci 2007;62:1375-1381.

10 Zoico E, Di Francesco V, Mazzali G, et al. High baseline values of fat mass, independently of appendicular skeletal mass, predict 2-year onset of disability in elderly subjects at the high end of the functional spectrum. Aging Clin Exp Res 2007;19:154-159.

11 Veronese N, Stubbs B, Trevisan C, et al. Results of an observational cohort study of hyperuricemia as a predictor of poor physical performance in the elderly: the Pro. V. A. study. Arthritis Care Res (Hoboken). 2017;69:1238-1244. https://doi.org/10.1002/acr.23118

12 Taaffe DR, Harris TB, Ferrucci L, et al. Cross-sectional and prospective relationships of interleukin-6 and C-reactive protein with physical performance in elderly persons: MacArthur studies of successful aging. J Gerontol A Biol Sci Med Sci 2000;55:M709-715.

13 Clouston SA, Brewster P, Kuh D, etal. The dynamic relationship between physical function and cognition in longitudinal aging cohorts. Epidemiol Rev 2013;35:33-50. https://doi.org/10.1093/epirev/mxs004

14 Sofi F, Valecchi D, Bacci D etal. Physical activity and risk of cognitive decline: a meta-analysis of prospective studies. J Intern Med 2011;269:107-117. https://doi. org/10.1111/j.1365-2796.2010.02281.x

15 Garcia-Pinillos F, Cozar-Barba M, Munoz-Jimenez M, et al. Gait speed in older people: an easy test for detecting cognitive impairment, functional independence, and health state. Psychogeriatrics 2016;16:165-71. https://doi. org/10.1111/psyg.12133

16 Wang Y, Singh S, Bajorek B. Old age, high risk medication, polypharmacy: a 'trilogy' of risks in older patients with atrial fibrillation. Pharm Pract (Granada). 2016;14:706. https:// doi.org/10.18549/PharmPract.2016.02.706

17 Wilson NM, Hilmer SN, March LM, et al. Associations between drug burden index and falls in older people in residential aged care. J Am Geriatr Soc 2011;59:875-880. https://doi.org/10.1111/j.1532-5415.2011.03386.x
18 Arnarson A, Ramel A, Geirsdottir OG, et al. Changes in body composition and use of blood cholesterol lowering drugs predict changes in blood lipids during 12 weeks of resistance exercise training in old adults. Aging Clin Exp Res 2014;26:287-292. https://doi.org/10.1007/ s40520-013-0172-0

19 Folstein MF, Folstein SE, McHugh PR. "Mini-mental state". A practical method for grading the cognitive state of patients for the clinician. J Psychiatr Res 1975;12:189-198.

20 Geirsdottir OG, Arnarson A, Briem K, et al. Effect of 12-week resistance exercise program on body composition, muscle strength, physical function, and glucose metabolism in healthy, insulin-resistant, and diabetic elderly Icelanders. J Gerontol A Biol Sci Med Sci 2012;67:12591265. https://doi.org/10.1093/gerona/gls096

21 ATS Statement. Guidelines for the Six-Minute Walk Test. Am J Respir Crit Care Med 2002;166:111-117.

22 Podsiadlo D, Richardson S. The Timed Up \& Go: a test of basic functional mobility for frail elderly persons. J Am Geriatr Soc 1999;39:142-148.

23 Martínez-González MA, Varo JJ, Santos JL, et al. Prevalence of physical activity during leisure time in the European Union. Med Sci Sports Exerc 2001;33:1142-1146.

24 Ainsworth BE, Haskell WL, Whitt MC, et al. Compendium of physical activities: an update of activity codes and MET intensities. Med Sci Sports Exerc 2000;32:s498-s504.

25 Paffenbarger RS, Wing AL, Hyde RT. Paffenbarger physical activity questionnaire. Am J Epidemiol 1978;108:161-175.

26 Caspersen CJ, Powell KE, Christenson GM. Physical activity, exercise, and physical fitness: definitions and distinctions for health-related research. Public Health Rep 1985;100:126-131.

27 Silva N, Menezes TN. The association between cognition and handgrip strength among the elderly: an integrative review. [Article in Portuguese]. Cien Saude Colet 2016;21:3611-3620. https://doi. org/10.1590/1413-812320152111.22872015

28 Tseng LA, Delmonico MJ, Visser M, et al. Body composition explains sex differential in physical performance among older adults. J Gerontol A Biol Sci Med Sci 2014;69:93100. https://doi.org/10.1093/gerona/glt027

29 Geirsdottir OG, Arnarson A, Ramel A, et al. Dietary protein intake is associated with lean body mass in communitydwelling older adults. Nutr Res 2013;33:608-612. https:// doi.org/10.1016/j.nutres.2013.05.014

30 Wells JL, Dumbrell AC. Nutrition and aging: assessment and treatment of compromised nutritional status in frail elderly patients. Clin Interv Aging 2006;1:67-79. 